\title{
Adeno-Associated Virus Gene Therapy for Liver Disease
}

\author{
Lisa M. Kattenhorn, Christopher H. Tipper, Lorelei Stoica, Deborah S. Geraghty, \\ Teresa L. Wright, K. Reed Clark, and Samuel C. Wadsworth* \\ Dimension Therapeutics, Cambridge, Massachusetts.
}

The field of adeno-associated virus (AAV) gene therapy has progressed rapidly over the past decade, with the advent of novel capsid serotype and organ-specific promoters, and an increasing understanding of the immune response to AAV administration. In particular, liver-directed therapy has made remarkable strides, with a number of clinical trials currently planned and ongoing in hemophilia A and B, as well as other liver disorders. This review focuses on liver-directed AAV gene therapy, including historic context, current challenges, and future developments.

Keywords: gene therapy, hemophilia, AAV, liver, clinical trials, metabolic

\section{INTRODUCTION}

TODAY, THERE IS AN INTENSE interest in developing gene therapy products for monogenic diseases, with the liver garnering significant attention due to the large number of conditions affecting hepatocytes and liver function. The scope of this review is limited to advances in gene therapy applications employing vectors based on adeno-associated virus (AAV), and the clinical and commercial applications of these vectors for the treatment of liver-based diseases. Multiple advances in the field have led to the continued refinement of AAV vector properties, the discovery of new versions of AAV capsids, and the completion of numerous robust therapeutic studies in relevant animal models of inherited diseases in animals, both small and large. To enable a full discussion of this field, the properties of the liver that make it amenable to AAV gene therapy and the properties of AAV vectors that make them especially relevant to liver gene therapy are reviewed. In addition, aspects of nonclinical studies that support clinical studies, as well as those that might offer insight into the field, are reviewed. Early and ongoing clinical trials are examined, attempting to draw some conclusions across the field, and, finally, the challenges for the broad clinical application of AAV gene therapy vectors for liver diseases are examined.
Much of the current enthusiasm for liver-directed AAV gene therapy product development stems from preclinical and clinical successes in the field of hemophilia B. Numerous studies in classic mouse and dog models of hemophilia A and B have demonstrated clear and robust long-term benefit from administration of AAV vectors encoding the relevant clotting factors, with the vector trafficking to the liver for gene expression. ${ }^{1-4}$ However, the path to clear clinical benefit for AAV hemophilia gene therapy has been a winding one. The first liverdirected clinical trial in hemophilia B, run by Manno et al., showed initial success, achieving remarkable factor IX (FIX) expression levels of $\sim 11 \%$ of normal, only to have levels subsequently return to the patient's baseline of $<1 \%$ of normal within weeks. ${ }^{5}$ The concomitant rise in liver enzymes stimulated the hypothesis that an immune response to the vector capsid was the culprit for the decline in expression and triggered an intense period of research focusing on immune responses to AAV.

A portion of the limitation of the Manno trial was placed on the vector capsid, AAV2, which in turn stimulated a period of concentrated development of alternative AAV capsids to overcome these AAV2 restrictions. After a 5-year clinical hiatus in the field, Nathwani et al. published the results of a

${ }^{*}$ Correspondence: Samuel C. Wadsworth, Dimension Therapeutics, 840 Memorial Drive, Cambridge, MA 02139. E-mail: sam.wadsworth@dimensiontx.com

( Lisa M. Kattenhorn, et al., 2016; Published by Mary Ann Liebert, Inc. This Open Access article is distributed under the terms of the Creative Commons Attribution Noncommercial License (http://creativecommons.org/licenses/by-nc/4.0/) which permits any noncommercial use, distribution, and reproduction in any medium, provided the original author(s) and the source are credited. 
study in which an AAV8 vector was able to deliver clinically meaningful levels of FIX to hemophilia B patients. The salient achievements of the Nathwani trial included demonstration of clinical benefit with restoration of as low as $\sim 2 \%$ of normal FIX expression, sustained expression of FIX for $>5$ years at $\geq 5 \%$, and the demonstration that intervention into the presumptive immune response with steroid administration could abort the increases in liver enzymes and preserve FIX expression. ${ }^{6}$

These encouraging results have sparked multiple AAV gene therapy trials for hemophilia B, as well as other liver and metabolic diseases. Additional improvements in delivery, capsid and vector genome designs, and better understanding of the immune response are building upon these encouraging results, and will potentially lead to treatments for other rare disorders.

\section{PROPERTIES OF THE LIVER}

The liver is a vital organ and plays an important role in human metabolism and other key physiologic functions. ${ }^{7,8}$ Hepatocytes, the most common type of liver cell, synthesize and metabolize a large number of proteins, including intracellular and secreted proteins responsible for a diverse range of critical functions in the body. ${ }^{9,10}$ These functions include carbohydrate use and storage, lipid metabolism, and hemostasis. In addition, the liver plays an important role in detoxifying naturally occurring metabolites such as ammonia and bilirubin. Numerous inherited metabolic disorders (IMDs) have their origin in the liver. Disruption of key metabolic pathways modulated by the liver can lead to the accumulation of toxic products and subsequent liver damage, or to the inappropriate or insufficient production of key proteins for proper metabolic function, both of which can have a range of deleterious systemic effects. Today, there are $>400$ described rare monogenic disorders associated with the liver. ${ }^{11-17}$

The liver also possesses unique anatomic properties that make it a preferred target for AAV-based gene therapy. ${ }^{18}$ Due to its unique dual blood supply, an adult human liver receives nearly $25 \%$ of the cardiac output of blood, filtering approximately $1 \mathrm{~L}$ of blood per minute, and accounts for $10-15 \%$ of the blood volume at any given moment. ${ }^{19}$ This means that administration of gene therapy systemically leads to rapid accumulation of high levels of vector particles within the liver. Specialized fenestrated endothelium along hepatic sinusoids allows for free passage of vector particles from the blood directly to the hepatocytes, making transduction of cells much more efficient than in organs with continuous en- dothelium (see Fig. 1). In part due to these anatomical features, the liver has the highest recorded AAV genome copies per tissue of natural isolates outside of primary immune organs. ${ }^{20,21}$

Because AAV vectors are largely non-integrating, an important property to consider with liver-directed gene therapy is the longevity of the transduced hepatocyte. In a fully developed liver, under normal conditions, $<1-2 \%$ of hepatocytes are turning over at any given time, while the rest remain in a quiescent state. ${ }^{14,15,22}$ In addition, turnover of mature hepatocytes occurs relatively slowly, with the average life span of non-resting hepatocytes ranging from 200 to 300 days. $^{16}$ Given the relative lack of hepatocyte turnover, gene therapy administered to these cells should experience minimal dilutional effects due to new hepatocyte generation. In the Nathwani study, published in the New England Journal of Medicine, hemophilia B patients are still experiencing durable FIX expression and clinical benefit for $>5$ years from the time of vector administration. ${ }^{6,23}$

The ideal goal of treatment for liver-associated monogenic diseases, including IMDs, is to revert a harmful or disease-causing genetic condition to its normal state. Substantial progress has been made in the treatment of these patients over the past six decades, beginning with the first report of dietary control of phenylketonuria in the early $1950 \mathrm{~s}^{24}$ However, many of these approaches are inadequate for long-term control. Monogenic diseases associated with the liver are particularly well-suited to gene therapy, as the nature of these diseases permits the use of well-described, and often clinically validated, biomarkers. Because gene therapy aims to revert an abnormal genetic condition toward a more normal state, the use of well-understood biomarkers can inform dose selection, enable evaluation of therapeutic efficacy, and serve as a surrogate for early signals of clinical benefit.

\section{AAV VECTORS IN GENE THERAPY}

There are several reasons why AAV vectors are useful for liver-directed gene therapy applications, especially when considering commercial drug development. An AAV vector consists of only two components: the protein coat, or vector capsid, and the vector DNA genome. Differences in the vector capsid are responsible for serological classification of vectors (i.e., AAV1 through AAV6) and structural classification of vectors with the advent of AAV7 and beyond. ${ }^{20,21}$ Despite differences in capsid protein amino acid sequence, all AAV capsids adhere to certain core structural themes, ${ }^{25}$ which to a large degree dictate the size and therefore the 


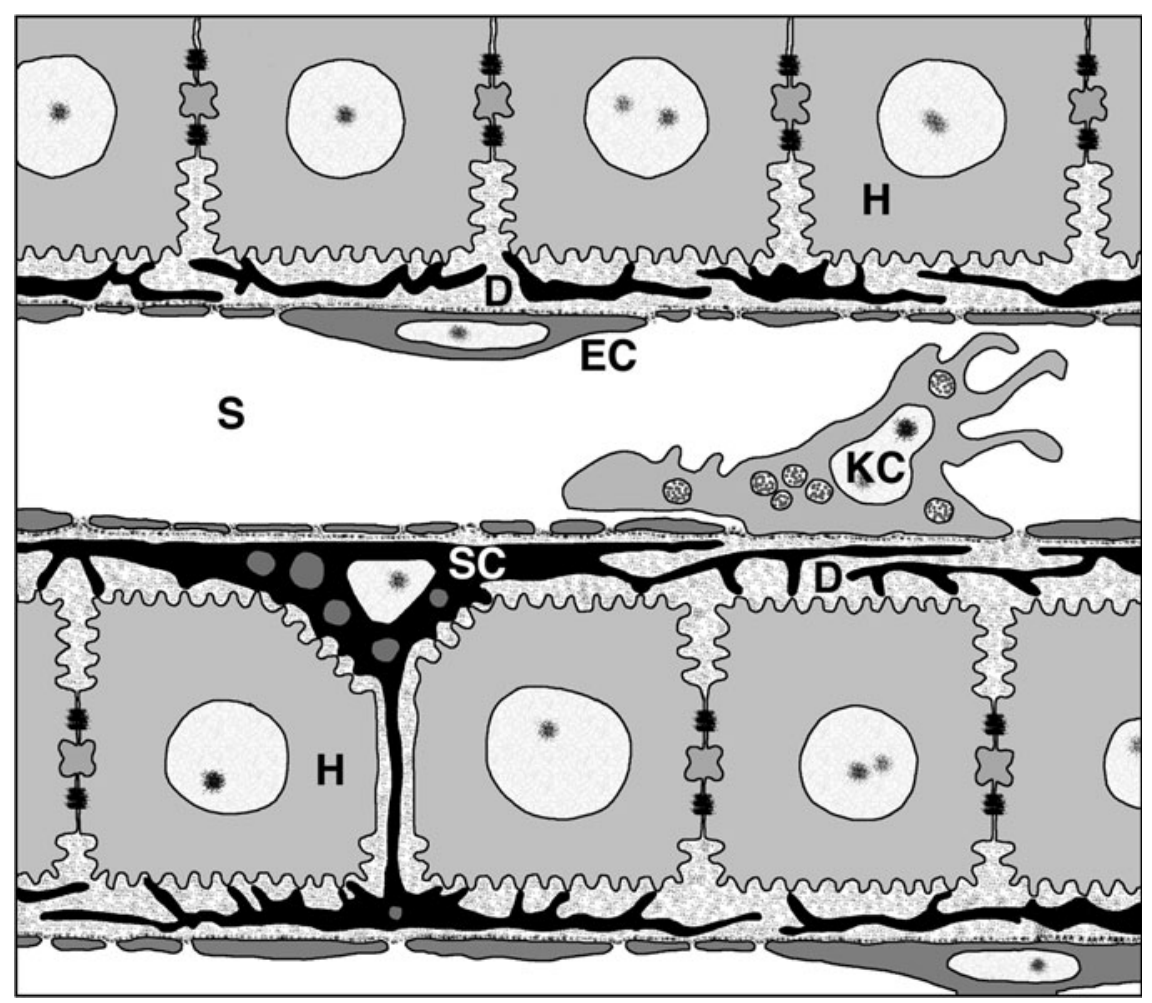

Figure 1. Architecture of the liver sinusoid. Liver sinusoids (S) are lined by fenestrated endothelia (EC) and interspersed Kupffer cells (KC), the resident macrophages of the liver. Stellate cells (SC), the major producers of liver ECM, are located inside the narrow space of Disse (D), which is formed by the sinusoidal cell layer and cords of hepatocytes $(\mathrm{H})$. Figure originally published in Frevert U, Engelmann S, Zougbédé S, et al. Intravital observation of plasmodium berghei sporozoite infection of the liver. PLoS Biol 2005;3:e192. DOI: 10.1371/journal.pbio.0030192.

DNA-carrying capacity of AAV vectors. Considering AAV vectors as biologic products, the conserved vector capsid structure and the inherent stability thereof offer advantages. Because no other proteins participate in the structure of the vector capsid, the analytical requirements for purity and identity of the final AAV vector product are simpler compared with other vector systems. The inherent stability of an AAV vector capsid also enables the application of robust downstream purification unit operations such as heat inactivation of potential adventitious agents, ion exchange chromatography, and various filtration and liquid handling operations that are standard within the biologics manufacturing realm. ${ }^{26-28}$

Using the well-studied AAV2 as the canonical representative, the AAV virus genome is limited to 4,679 nucleotides with 290 nucleotides reserved for the ITRs, the latter being the only cis-acting requirement for packaging of an AAV vector genome within the capsid. ${ }^{29}$ Thus, in the ideal case, the carrying capacity of an AAV vector genome, including transcriptional control sequences, any $5^{\prime}$ and $3^{\prime}$ mRNA untranslated sequences, protein (or RNA) coding sequences, and poly-A addition signals is limited to approximately 4,400 nucleotides. Therefore, in each case, the precise nucleotide sequence of the AAV vector is known and analytical tools exist to enable sequencing of the plasmids entering the manufacturing process and of the AAV vector that emerges as the final purified product. For large protein coding sequences, there are stringent size limitations on each of the noncoding elements within the AAV vector genome, and attempts to produce oversized vectors have resulted in inefficient packaging and truncated genomes. ${ }^{30,31}$ To overcome the size limitation challenge, dual vector systems have been proposed, where the larger genome would be recreated through recombination of the partial genomes. ${ }^{32}$ In addition, the effort to develop cassettes for factor VIII (FVIII) expression serves as an object lesson in setting expectations for both efficacy and quality of large-genome rAAV. Bearing in mind the well-established packaging "cliff" of approximately $5.2 \mathrm{~kb}$, success has only come after considerable effort from individual groups to minimize the size of the protein itself (deletion of the B-domain), establish the bare minimum regulatory sequences required for a reasonable level of tissue-specific expression, and test alternate serotypes for greater size tolerance. ${ }^{31,33-35}$ For small protein coding sequences, there is ample space within an AAV vector genome, but the opposite problem, having a vector genome that is too small, 
can also occur. Engineering a vector genome that is too small can lead to inclusion of the dimeric replication intermediates, adjacent plasmid sequences, or higher levels of host cell DNA. ${ }^{36}$ Taken together, the above considerations indicate that an AAV vector product can be manufactured and characterized as a well-controlled biologics product, employing systems that are currently established within the biologics manufacturing world.

\section{CHOICE OF SEROTYPE}

Given the anatomical features of the liver reviewed in the introduction, it might seem that achieving effective liver transduction following systemic delivery would be serotype independent. However, it was apparent from early clinical trials seeking to deliver FIX that improvement in AAV2 was needed, which stimulated the development of new AAV capsids for clinical use.

In 2002, the serotype AAV8 was discovered in rhesus macaques. ${ }^{37}$ Reports from multiple groups confirmed that AAV8, a member of the Clade E family of AAV, outperformed AAV2 and others in liver transduction and expression, demonstrating that serotype choice plays a significant role in successful transgene delivery. ${ }^{37-39}$ Key advantages of vectors based on AAV8 and other members of the Clade E family include the ability to achieve a high level of hepatocyte gene transfer via peripheral vein administration, presence of a lower proportion of neutralizing antibodies in the human population, and commercially useful manufacturing properties such as high yield and release of vector into the cell culture medium. ${ }^{40,41}$ The research that led to the early clinical development of AAV8 vectors culminated in the aforementioned successful clinical studies by Nathwani et al., in which all hemophilia B subjects in the trial appeared to derive clinical benefit from the experimental gene transfer protocol. Importantly, a smaller proportion of potential subjects failed the neutralizing antibody screening test, allowing for more facile patient enrollment. The peripheral vein administration route was successful in delivering the AAV8 FIX vector to the liver, and the elicitation of ALT after vector infusion was reduced and readily controlled by a course of prednisone treatment. ${ }^{6}$

Concurrent with ongoing searches for totally new AAV capsids, many groups have sought to engineer current serotypes to improve liver transduction and/ or avoid preexisting immunity. These include alteration of surface residues to reduce phosphorylation and improve trafficking, peptide insertion, ligand modification, and so-called "directed evolu- tion” or capsid shuffling. ${ }^{42-47}$ One engineered serotype, LK03, was identified using the latter method in the context of a rodent model in which mouse livers were repopulated with human hepatocytes. ${ }^{48}$ Reports on the in vivo transduction superiority of LK03 versus AAV8 and AAV3B are mixed, probably due to the models used. Regardless, LK03's most promising characteristics may be that it seems to be more resistant than AAV2 and AAV3B to pooled human IVIG, a surrogate for preexisting neutralizing antibodies in the human population, and therefore may be available to a greater number of patients. ${ }^{48}$ However, noting that LK03 shares the majority of its variable domains with AAV3B (and HGFR receptor usage), this conclusion is also in dispute. Wellstructured trials may be needed to resolve the benefits of the current generation of engineered capsids.

\section{LIVER-SPECIFIC PROMOTERS}

A future goal of AAV vector capsid engineering is eventually to limit gene transfer solely to the organ of interest, in this case the liver. Until this goal is fully realized, however, efforts must focus on the expression of the therapeutic transgene, which should largely be limited to hepatocytes to improve safety and efficacy profiles. A rich body of work has been generated on liver-specific promoters (LSPs), from deconstructed single-locus cassettes defined by "promoter bashing," to modern chimeric cassettes, to next-generation cassettes identified by deep sequencing techniques and high-throughput screening, and finally to transgene-specific regulation.

Early LSPs drew directly from work aimed at understanding the regulation of high abundance liver secreted proteins such as human serum albumin and alpha-1-antitrypsin. ${ }^{49,50}$ Seeking to tailor and control transcriptional activity further, chimeric cassettes were developed. These LSPs are essentially strings of modular building blocks that have been identified and characterized independently. Among the best-known examples of chimeric promoters are those that have been used in the hemophilia A and B trials. Those used by Kay et al. and Nathwani et al. began as a combination of the Apolipoprotein E/C-I hepatic control region combined with the human alpha-1-antitrypsin core promoter. ${ }^{51-54}$ At $754 \mathrm{bp}$, this combination, though potent, proved to be too large to fit into a self-complementary vector. What followed was a stepwise reduction in LSP size to first accommodate self-complementary $F I X$, called LP1, and even smaller to drive the expression of FVIII, called HLP, based upon closer analysis of the two core elements. ${ }^{55,56}$ The cassette used by the Verma et al. and Wilson et al. groups uses a different set of mod- 
ules, specifically two copies of alpha 1 microglobulin/ bikunin enhancer coupled to the core promoter of human thyroxine-binding globulin (TBG). Expression is further stabilized by the inclusion of a woodchuck hepatitis virus post-transcriptional regulatory element (WPRE). ${ }^{57-60}$ Finally, Naldini et al. made their promoter, designated "ET," and described as randomly assembled hepatocyte-specific transcription factor binding sites linked to the murine transthyretin promoter. ${ }^{61}$ Though it does not drive expression as well as those that entered clinical trials, it presages next-generation cassette design, exemplified by an alternative approach employing in silico analysis of microarray data to identify liver-specific control elements. Interestingly, this approach re-identified the core enhancer of alpha-1antitrypsin, suggesting that another avenue for engineering tissue-specific gene expression exists. ${ }^{62}$

\section{CODON OPTIMIZATION}

While restriction of gene expression to hepatocytes is critical, equally important is the property of prolonged gene expression from a single dose of vector. Prolonged gene expression, meaning expression at therapeutic levels over a time span measured in years, is a key part of the value proposition for AAV liver-directed gene therapy. The data from large animal studies with AAV vectors, particularly in hemophilia dog models, suggest that multi-year therapeutic benefit, perhaps $\geq 10$ years, can be achieved with a single vector dose. ${ }^{63}$ Fortunately, the clinical data available to date indicate that prolonged therapeutic benefit to hemophilia $B$ patients can also be achieved with a single vector dose. ${ }^{6}$ Obviously, more clinical results are needed before firm general conclusions can be drawn regarding the duration of therapeutic benefit from a single dose.

While selection of enhancer and promoter elements seeks to endow AAV vectors with hepatocyte selective expression, the high vector dose requirements of liver-directed gene therapy make it useful to attempt to maximize the level of gene expression per dose. Transgene codon optimization is an option to maximize expression and therapeutic potential. Though the candidate selection process for transgenes is often not reported, codon optimization appears to be the default for most vectors used in research studies and clinical trials. ${ }^{64-67}$ Briefly, the goal of codon optimization is to match the codon usage in the target therapeutic transgene with the abundance of transfer RNA (tRNA) for each codon in a particular organism and/or cell type. There are examples where this process can result in a significant increase in gene expression, ${ }^{68}$ presumably by increasing the overall rate of translation via eliminating pauses created by codons that utilize lowabundance tRNAs. Codon optimization applied indiscriminately could potentially be detrimental versus relying on native sequence. Many proteins have complex structural topologies, where translational pauses might allow proper folding and/or interaction with translocation machinery; eliminating these pauses could lead to misfolding and loss-offunction, or aggregation. ${ }^{69,70}$ However, as hepatocytes function to synthesize and secrete massive levels of protein on a continuous basis, this potential limitation may be less of a concern for liver-directed therapies.

\section{MANUFACTURING CHALLENGES FOR AAV LIVER-BASED GENE THERAPY}

The challenges of manufacturing AAV vectors at a scale and quality level appropriate for clinical trials held back product development for many years. However, significant improvements have been made in recent years, enabling the current expansion of the number and spectrum of clinical trials employing AAV vectors. ${ }^{71-73}$

Clinical and eventual commercial manufacturing of AAV vectors for liver-based gene therapy products is an especially daunting challenge due to the large dose requirements required for systemic administration to reach the desired hepatocytes. Comparison of recent gene therapy programs illustrates the challenge of liver-based gene therapy versus other targets for vector delivery. A clinical gene therapy for Leber congenital amaurosis type 2 (LCA2), for example, utilized doses of approximately $6 \times 10^{10} \mathrm{vg}$ of recombinant AAV2 vector, altered to carry the human RPE65 gene (rAAV2-CBSB-hRPE65) in a volume of $150 \mu \mathrm{L}$ per eye. ${ }^{74}$ In contrast, the high dose of recombinant AAV8 encoding FIX used in the Nathwani clinical trial used doses of $2 \times 10^{12} \mathrm{vg} / \mathrm{kg}$, or $1.61 \times 10^{14} \mathrm{vg}$ for the average $80.7 \mathrm{~kg}$ patient in the trial. Thus, the amount of vector for a single dose by Nathwani was on average 271 times higher than for the LCA2 trial for a single eye, or 135 times higher for both eyes. Similar differences are relevant for ear, brain, or other local only treatments versus systemic delivery for liver-directed therapies. To meet these high dose requirements for liver-directed gene therapy, suspension bioreactor platforms are under development. ${ }^{72,73,75-78}$

\section{VECTOR DISTRIBUTION IN NONCLINICAL MODELS}

The distribution and transgene expression of several vectors after intravascular delivery has been studied in mice, rabbits, dogs, and nonhuman 
primates. The overall tissue distribution between various nonclinical species is generally similar. In mice, AAV8 has been demonstrated to transduce liver efficiently, with levels of transduction 10-100 times greater than early generation AAV2 vectors, and approximately 9- and 16-fold higher than AAV7 and AAV5, ${ }^{37}$ respectively. This is consistent with the superior liver-transducing properties of Clade $\mathrm{E}$ $\mathrm{AAV}$ vectors. In a subsequent study in mice, a targeted group of liver-specific vectors were evaluated (AAV8, AAV6.2, AAV7, AAVrh.64R1, AAVhu.37, AAVrh.8, AAVrh.32.33), with biodistribution to liver and non-hepatic tissues as a criterion. Of the vectors tested, AAV8, AAVrh.8, and AAVhu.37 demonstrated the greatest future potential based on liver transduction and durability of expression. ${ }^{39}$ In terms of biodistribution to extrahepatic tissues (heart, lung, kidney, spleen, pancreas, brain, muscle, and testes), AAV6.2 and AAVrh.32.33 had the highest levels of extrahepatic vector, with AAV8, AAV7, AAVrh.64R1, AAVhu.37, and AAVrh.8 having similar levels of significantly lower levels in nonliver tissues.

There are a limited number of studies that directly compare results of AAV vectors between rodents and non-rodents, but these can provide important information to understand differences between species and potential allometric scaling. The biodistribution to liver and expression from AAV2, AAV5, AAV6, and AAV8 vectors encoding FVIII were evaluated in hemophilia A mice and dogs. ${ }^{4}$ Again, AAV8 had dramatically higher levels of transduction in the livers of mice, approximately twofold higher than AAV6, sevenfold higher than AAV2, and 100-fold higher than AAV5. The relative expression of hFIX between vectors showed a similar pattern, albeit with a slightly reduced magnitude of difference between serotypes. In comparison, in hemophilia A dogs, the levels of transduction and expression of canine FVIII was similar between each vector, each producing approximately $2-5 \%$ of normal canine FVIII activity, in contrast to the results in mice, which varied from $20 \%$ for AAV2, $50 \%$ for AAV6, and $110 \%$ for AAV8.

In preclinical work for hemophilia B, AAV8 also produced the highest level of FIX in C57BL/6 mice, approximately 17- to 84-fold higher than AAV2 and AAV5 vectors encoding hFIX after administration of a given dose, with the same pattern of transduction levels for each vector. ${ }^{52}$ In rhesus monkeys, animals treated with AAV8 had levels of hFIX that were approximately twofold higher than AAV5 at steady state. ${ }^{52}$

In both dogs and monkeys, the differences in transduction efficiency between different serotypes is less pronounced than in mice, with a lower transduction efficiency overall. The partitioning of vector in tissues of AAV8 in rhesus monkeys is similar to that of mice and rabbits, however, with the liver having the highest level, at least 100-fold more than other tissues, ${ }^{79}$ and vector detected in all tissues examined, including the brain, testes, muscle, kidneys, lungs, heart, and spleen (in ascending order). ${ }^{80}$ A robust data set comparing the efficiency of gene transfer of the same AAV vector product between monkeys and humans is lacking, but data from ongoing clinical trials should begin to fill this gap.

\section{LOCALIZATION OF HEPATOCYTE GENE TRANSFER}

Although nonclinical and now clinical experiences in hemophilia offer important insights into liver gene therapy of secreted proteins, gene therapy for non-secreted proteins brings about a separate set of preclinical challenges. As mentioned previously, transduction rates and transgene expression can vary between species, and so can the site of vector predilection of cell transduction within the liver. In a 2011 paper, Bell et al. compared the expression pattern of AAV8 expressing green fluorescent protein (GFP) in liver between mice, dogs and nonhuman primates. They demonstrated a predominantly pericentral expression of GFP in both murine and canine liver, regardless of whether a liver specific promoter (TBG) or a ubiquitous promoter (CMV) was used. ${ }^{81}$ The expression pattern was also independent of the site of vector administration, with pericentral expression occurring in dogs after injection in either the cephalic vein or the hepatic artery. Transduction within the liver of adult rhesus and cynomolgus macaques, in contrast, occurred mainly in periportal regions. Interestingly, livers from mice and rhesus monkeys treated with AAV8 GFP as neonates or infants showed a more uniform distribution of GFP expression, lacking a predilection for either periportal or central venous areas. Transduction of neonatal animals, however, often leads to loss of transgene expression over time, due to the diluted effects of replicating hepatocytes. ${ }^{82} \mathrm{~A}$ more recent paper demonstrated that localization in mice can be capsid dependent, with AAV2 localizing to periportal regions, while AAV 7, 8, 9, and rh10 localized to pericentral regions, as seen previously. ${ }^{83}$

The reason for the differences in localization between species is not known. However, it is speculated that it may be due to receptor localization and abundance, presence or absence of Kupffer cells, or differences in sinusoidal width between species. ${ }^{81}$ It is interesting to note that both hydrodynamic 
injection of plasmids, as well as injection of dyes such as Evans Blue, result in accumulation of these substances within central venous regions of murine livers. ${ }^{81}$ The localization of these compounds within nonhuman primate livers is not known. Further studies are needed to understand the mechanism of localization for these viral vectors in animal models and how they compare to human patients.

Although less important for the production of secreted proteins such as FIX or FVIII, localization of vector transduction within the liver may be significant in the treatment of inborn errors of metabolism. Using gene therapy for restoring metabolic function, as in the case of ornithine transcarbamylase deficiency (OTC) or methylmalonic acidemia (MMA), is well summarized in the 2008 review by Alexander et al. ${ }^{84}$ Treating these disorders in human patients will likely require a higher percentage of transduction of hepatocytes than for secreted proteins, as well as careful consideration of hepatocyte zonality. It is well known that the liver is divided into functional zones, and although the secretion of many proteins from hepatocytes does not appear to be zonally specific, many metabolic processes, including ammonia detoxification, are localized to specific regions of hepatic architecture. ${ }^{85}$ The effects of enhancing or disrupting this pattern on restoring metabolic function will need to be further explored in the context of AAV gene therapy as these treatments move toward the clinic.

\section{PRECLINICAL IMMUNE RESPONSES TO AAV GENE THERAPY}

As mentioned in previous sections, early work with AAV in liver-directed transduction was hindered by unexpected immune responses to AAV, namely in the form of neutralizing antibodies and cytotoxic T-cell responses to the AAV capsid that were not predicted by preclinical studies, highlighting one of the major preclinical challenges when working with AAV. To date, an animal model completely predictive of the immune response seen in humans to this virus has yet to be discovered.

Both mice and nonhuman primates have proven to be successful models for the effect of neutralizing antibodies (NAbs) on transduction. Studies in both species have demonstrated that NAb titers as low as 1:5 can block AAV vector transduction of the liver completely, and that vector remains susceptible to antibody-mediated neutralization for several hours after intravascular delivery. ${ }^{86,87}$ Due to the strong conservation in capsid proteins between AAV serotypes, further work needs to be done to examine the cross-reactivity of antibodies and the potential for re-dosing patients with alternative serotypes in the future.

Although animal models predicted many aspects of the human immune response to transgene products, they largely failed to predict responses to AAV capsid. The first indication that human $\mathrm{CD} 8^{+} \mathrm{T}$ cells could limit efficacy of gene transfer was uncovered in the initial trial of AAV gene transfer to liver for hemophilia. ${ }^{5}$ In this trial, AAV2 was used to deliver human FIX under the control of a liver-specific promoter. Initially, the first subject infused at the high dose, $2 \times 10^{12} \mathrm{vg} / \mathrm{kg}$, expressed FIX at levels in the range of $10-12 \%$, concurrent with what had been seen in the dog model of hemophilia. After a period of several weeks, however, FIX expression disappeared, accompanied by a self-limited and asymptomatic transaminase elevation in the blood. This series of events, not observed in the experimental animal models, spurred a large-scale effort to determine the underlying cause of this loss of expression. Ultimately, it was hypothesized that declining transgene expression was due to anti-capsid cytotoxic T-cell mediated destruction of transduced hepatocytes displaying capsid antigen via major histocompatibility complex class I pathways.

Multiple efforts have been made to develop preclinical models of the human immune response to the AAV capsid. In early studies, mice were immunized with AAV capsid protein or viral vector and then exposed to AAV expressing FIX. Despite the presence of cytotoxic $\mathrm{CD}^{+} \mathrm{T}$ cells specific for the AAV capsid protein within the liver, hepatocyte production of FIX was preserved to the same levels as seen in naive mice. ${ }^{88}$ Further studies in mice confirmed that although AAV did induce a capsidspecific cytotoxic $\mathrm{CD} 8^{+} \mathrm{T}$-cell response, transduced hepatocytes did not become cytolytic targets. ${ }^{89}$ Recent attempts to boost this immune response in animals, including the use of adjuvants ${ }^{90}$ or incorporation of SIINFEKL peptide epitopes into the AAV vector capsid concurrent with the use of OT-1 mice, ${ }^{91}$ have recapitulated some of the immune response, but the utility and relativity of such contrived circumstances to administration in humans is questionable. Work done in nonhuman primates has not met with any additional success, and researchers have been unable to mimic reliably the cytolytic T-cell response and subsequent rise in alanine aminotransferase and aspartate aminotransferase to date. The reason for these speciesspecific differences is unknown. However, speculation suggests it may be due to the loss of a subset of inhibitory immunoglobulin-like lectins on human $\mathrm{T}$ cells during evolution. ${ }^{92}$ Further proof of this remains to be demonstrated, however. 


\section{LIVER-MEDIATED TOLERANCE}

Although the liver is responsible for making many pro-inflammatory components of the immune system, it also plays a critical role in inducing tolerance to dietary and self-antigens. This tolerance induction is especially intriguing in the context of liver-directed gene therapy. Pastore et al. first described the phenomenon of limitation of immune responses to an otherwise immunogenic protein by use of a promoter sequence derived from a liverspecific gene. ${ }^{93}$ Direct comparison of two adenovirus vectors expressing human alpha 1-antitrypsin (hAAT) from the ubiquitous mouse phosphoglycerate kinase 1 (mPGK) promoter or from the liverspecific albumin promoter indicated that $\mathrm{C} 3 \mathrm{H} / \mathrm{HeJ}$ mice produced antibodies to hAAT when it was expressed from the $\mathrm{mPGK}$ promoter but not when it was expressed from the albumin promoter. ${ }^{93}$ Since then, a number of studies have demonstrated the induction of tolerance to different proteins following in vivo hepatocyte transduction and transgene expression. Work by Cao et al. demonstrated a role for regulatory $\mathrm{T}$ cells in liver tolerance. ${ }^{94}$ The authors used Rag2 ${ }^{-1-}$ BalbC mice transgenic for a T-cell receptor reactive against a peptide from chicken ovalbumin (OVA). These mice lack regulatory $\mathrm{T}$ cells normally. However, upon administration of AAVOVA, they develop tolerance to the OVA transgene, accompanied by formation of regulatory $\mathrm{T}$ cells expressing $\mathrm{CD} 4^{+} \mathrm{CD} 25^{+} \mathrm{FOXP}^{+}$. A role for regulatory $\mathrm{T}$ cells in induction of tolerance has been demonstrated in nonhuman primates as well. A paper from Mingozzi et al. demonstrated that the administration of the anti-interleukin-2 receptor antibody daclizumab, in the context of an immunosuppressive regiment in nonhuman primates administered AAV-FIX, led to decreased tolerance of the transgene versus mycophenolate mofetil (MMF) and sirolimus alone. ${ }^{95}$ The authors went further to discover that daclizumab inhibited the formation of $\mathrm{CD} 4^{+}$ $\mathrm{CD}^{2} 5^{+} \mathrm{FOXP}^{+} \mathrm{T}$ cells, which are essential to the development of liver tolerance to FIX. ${ }^{95}$

In addition to regulatory T cells, Kupffer cells and liver sinusoidal endothelial cells (LSECs) have also been shown to contribute to development of tolerance, potentially by the secretion of immunomodulatory factors such as nitric oxide and IL-10. ${ }^{96,97}$ Mouse LSECs function as a specialized form of antigen-presenting cell and express receptors that promote antigen uptake, including the mannose receptor and the scavenger receptor, and molecules that promote antigen presentation, including CD40, CD80, and CD86. Although mouse LSECs can prime both CD4 and CD8 T cells, the most common consequence of this is tolerance induction versus activation. ${ }^{96}$ The reasons for this are still unclear, as is whether this translates to human LSECs. What is clear, however, is that the presence of specialized $\mathrm{T}$ cells, in conjunction with Kuppfer cells and LSECs, establishes a unique locally suppressive microenvironment, contributing to the development of tolerance to transgenes expressed in the liver. ${ }^{98,99}$

Although transduction of the liver versus other organs may lead to advantages in establishment of tolerance to gene therapy vectors and transgene production, ${ }^{100,101}$ considerations of transgene immunity are still necessary, especially in cases of proteins of high immunogenicity, such as FVIII. The immunogenicity of a delivered transgene depends on a number of factors, including vector selection, route of administration, targeted transgene expression levels, and the genotype of the host. ${ }^{102}$ Despite the complexity of attempting to suppress immune responses to therapeutic transgenes while avoiding unwanted side effects, pharmacological interventions are still being pursued. Sack et al. explored the use of B-cell depletion in a model of AAV mediated $F$ VIII gene delivery with some success, although the level of FVIII expression from different vectors was a confounding factor. ${ }^{103}$ Other pharmacological interventions include cyclophosphamide in a null mutant canine model of hemophilia $\mathrm{B},{ }^{104}$ as well as cyclophosphamide and methylprednisolone in murine models of hemophilia B. ${ }^{105}$ Immune responses to therapeutic proteins are not limited to gene therapy, in that development of anti-drug antibodies is also an issue in the protein therapeutics realm. Ellinwood et al. successfully employed a combined immunosuppression regime of mycophenolate mofetil plus cyclosporine to treat Sanfilippo and Hurler disease in affected dogs carrying null mutations of the relevant genes, and a relatively short course of a widely prescribed immunosuppressive drug, methotrexate, has been successfully employed in animal models of Pompe disease and in Pompe patients. ${ }^{106-108}$ Through studies such as these, an armamentarium of immunosuppressive drugs and protocols potentially useful in the context of gene therapy is being assembled and may prove useful in future gene therapy work.

\section{REMAINING CLINICAL CHALLENGES FOR LIVER-BASED AAV GENE THERAPY}

Assessment of the remaining challenges for broad commercial application of AAV gene therapy vectors across the full spectrum of liver-based diseases can clearly be debated. Two commonly discussed challenges have been selected for discussion 
below: re-administration of vector and risk of hepatocellular carcinoma.

\section{AAV vector re-administration}

It is well-appreciated that systemic administration of AAV vectors to seropositive individuals will be blocked by the presence of neutralizing antibodies in subjects. ${ }^{109,110}$ It is therefore understood that simple re-administration of the same AAV vector product will not be successful due to blocking neutralizing antibodies, and there are ample data to suggest that re-administration of closely related AAV vector products will also not be successful. ${ }^{111}$ Nonetheless, the issue of vector re-administration is an important one and is the subject of active research. Three different avenues for re-administration have been published: capsid switching, removal of antibodies from serum, and administration of decoys to adsorb antibodies. An example of capsid switching relevant to the discussion of hemophilia gene therapy was published by Wang et al. ${ }^{59}$ In this study, hemophilia B dogs were given an intravenous dose of AAV2 FIX that resulted in modest therapeutic benefit. To test the capsid switch re-administration hypothesis, a second dose of an AAV FIX vector, this one packaged in an AAV8 capsid, was given. This second dose resulted in a dramatic improvement in FIX expression and correction of the bleeding disorder, confirming that it is possible to re-dose animals with alternative serotypes of AAV.

Antibody removal or reduction to enable AAV re-administration was tested using the process of plasmapheresis. ${ }^{112}$ These studies were conducted in rhesus macaques with AAV vectors encoding a microdystrophin gene. Two cycles of plasmapheresis were shown to reduce serum antibodies in animals with high titers to levels equivalent to animals in which only low levels of antibodies were detected. Importantly, gene transfer to muscle was blocked in animals with high antibody titers but was successful in animals that had undergone the plasmapheresis procedure.

Finally, Mingozzi et al. confirmed the ability of co-administration of empty AAV capsids to serve as decoys to block the action of existing neutralizing antibodies temporarily. ${ }^{113}$ A large excess of empty capsids ( $\sim 100$-fold) was able to allow gene transfer to the liver with the cognate capsid while also not blocking gene transfer by competition with cell surface receptors. The same results were obtained in cell-based assays and in vivo in mice and monkeys. Obviously each of these potential avenues for vector re-administration will have to be confirmed in clinical studies before coming into general use.

\section{Risk of hepatocellular carcinoma}

Multiple studies have been conducted examining the potential effects of AAV integration on the development of hepatocellular carcinoma (HCC), a correlation currently hotly debated within the gene therapy community. This potential risk was first highlighted by Donsante et al. in 2001, utilizing recombinant AAV (rAAV) gene therapy to treat mucopolysaccharidosis type VII in newborn betaglucuronidase (GUSB) deficient mice. ${ }^{114}$ The authors reported an increased incidence of HCC in mice treated with rAAV compared with untreated controls. Their initial conclusion, based on quantitative polymerase chain reaction analysis of tumor samples, was that tumorigenesis was unlikely related to integration of rAAV due to the low numbers of vector genome copies in tumor tissue. However, a follow-up study of tumors from these mice identified four examples of AAV integration sites within the delta-like homologue 1-deiodinase type 3 (Dllk1Dio3) region. ${ }^{115}$ Two of these insertions were within MIR341, an insertional location mirrored in later studies as well. ${ }^{116}$ It is important to note that there is no human orthologue to MIR341. However, upregulation of other transcripts within the orthologous Rian region in humans have been implicated in a poor prognosis for patients with HCC. ${ }^{117}$

A study conducted in 2006 on the utility of gene therapy in the treatment of ornithine transcarbamylase (OTC) deficiency examined the frequency of $\mathrm{HCC}$ in spf ${ }^{\text {ash }}$, a model of this disease, and B6C3F1 control mice. Animals were treated with various AAV serotypes (2, 7, 8, and 9) encoding murine OTC (mOTC) or a control vector, AAV2/8-LacZ. ${ }^{118}$ All experimental groups developed liver nodules. However, mice that received AAV with mOTC as the transgene showed lower levels of nodules than either untreated control animals or animals treated with AAV-LacZ. This suggests that administration of AAV-OTC and correction of metabolic abnormalities with gene therapy may have actually been protective against the development of HCC in this mouse strain. This would not be surprising considering the metabolic alterations that occur in hepatocytes with disrupted urea cycles.

In a follow-up study, liver nodules or tumors from these same animals receiving AAV-mOTC, as described above, were collected and analyzed for integration sites. ${ }^{119}$ Mice were chosen with the highest numbers of vector DNA copies in the liver, and integration sites were compared between tumor and normal liver sections from five animals. Integration sites were distributed along the length of each chromosome without obvious clustering in hot spots. Integration sites were generally enriched 
in gene-dense regions and near gene $5^{\prime}$ ends, compared with computationally random distributions. A total of five sites in the Dlk1-Dio3 region could be verified, one from normal tissue and four from tumor-nodule tissue. In all four cases, integration was closest to the Rian locus.

A recent paper from Chandler et al. further examines the role of AAV integration in the formation of HCC in mice. ${ }^{116}$ Similar to previous studies, it was demonstrated that AAV most frequently integrates into highly expressed genes, including albumin and a-fetoprotein. Integration of AAV into the Rian locus was the only integration site associated with increased development of hepatocellular carcinoma, and only under a restricted set of conditions. First, HCC formation was dose dependent. A statistically significant increase in HCC formation was observed at $1 \times 10^{14} \mathrm{GC} / \mathrm{kg}(84 \%)$, but not at doses $\leq 7 \times 10^{12}$ GC/kg (12\%). Second, formation of HCC was highly dependent on the age at inoculation. Neonatally inoculated mice developed increased HCC, but mice inoculated at $\geq 6$ weeks of age did not develop tumors above background. This is consistent with previous studies, which found increased risk of HCC in neonatally inoculated mice ${ }^{114}$ but not in older mice. ${ }^{120}$ Third, development of tumors appeared to be related to specific promoter/enhancer combinations. There was a statistically significant increase in tumorigenesis with AAV vectors encoding the TBG or chicken B-actin enhancer and promoter combination when compared with other promoters, including a-1 antitrypsin (AAT). Similar to previous studies, the majority of integration sites within Rian were located in MIR341, a gene lacking an orthologue in humans. No microhomology between any $\mathrm{AAV}$ vector genome and the integration sites in MIR341 could be detected, making it difficult to translate this finding to the human genome. In this study, there was no statistically significant difference in the incidence of HCC between AAV8 containing the transgene of interest (MUT) when compared to AAV8 encoding GFP. These results, along with similar data from other publications, ${ }^{120}$ indicate that the development of HCC is likely not associated with a particular transgene, although integration specificity due to transgene sequence cannot be ruled out.

Translation of data from mice to humans can often be difficult. A recent publication demonstrated an association between wild-type (wt) AAV2 insertional mutagenesis, the dysregulation of neighboring genes, including cancer driver genes CCNA2, TERT, CCNE1, TNFSF10, and KMT2B, and development of HCC in humans. ${ }^{121}$ The authors demonstrated the presence of wtAAV2 sequences within
11/193 human HCC biopsies. The relationship of the presence of AAV DNA to the development of carcinoma is not clear, however, as 5/11 biopsies were from patients with other well-known HCC driving conditions, including alcoholism and hepatitis B and $\mathrm{C}$ infections. An additional four biopsies presented with AAV-unrelated mutations that have previously been linked to HCC formation. Therefore, only two biopsies contained fragments of wtAAV2 sequences with no other clear driver for mutagenesis. In addition, more non-tumor samples were positive for AAV2 DNA than tumor samples in this study. However, the number of recovered reads in tumor tissue was higher. Unlike in the murine studies, no integration was noted at or near any gene within the Rian locus. This suggests that the specific locations for integration of AAV into the human genome likely vary from the locations within the mouse genome. The author's conclusion that AAV is associated with an increased risk of HCC is hotly disputed, with a number of rebuttals quickly following publication, arguing that the presence of this ubiquitous virus is simply association and not causation. ${ }^{122,123}$

The relevance of this study to the safety of AAVmediated gene therapy is not clear, as $30-50 \%$ of the human population is seropositive for AAV2 and yet AAV2-induced hepatocellular carcinoma is not widespread, and only a small fraction $(5.7 \%)$ of the examined HCCs were positive for AAV2 in this study. ${ }^{41,121}$ When comparing data between wtAAV and rAAV vectors, two things must be considered. The first is that rAAV vectors lack wild-type rep proteins, and although it has been demonstrated that integration can still occur, it is at lower frequency compared with wild-type viruses. ${ }^{124}$ The second is that conversion of wtAAV to $\mathrm{rAAV}$ vectors involves the deletion and rearrangement of portions of the viral genome to make room for insertion of transgenes. Recent unpublished work from the lab of Ian Alexander demonstrates the presence of strong promoters within wtAAV2 ITRs, in regions that are largely absent from many AAV gene therapy vectors.

Unfortunately, the total risk of development of hepatocellular carcinoma secondary to AAV gene therapy in humans is still unknown, but, to date, no genotoxic events have been reported in the $>130$ rAAV clinical trials conducted so far. ${ }^{125,126}$ Kaeppel et al. performed integration site analysis in the muscle of patients from their lipoprotein lipase trial and discovered very low integration frequency and random nuclear integration. ${ }^{127}$ Additionally, a recent publication by Gil-Farina et al. examining liver samples from nonhuman primates in nonclinical studies and human patients in a clinical trial for acute intermittent porphyria demonstrates 
that although rAAV did integrate into the human genome, it did so with extremely low frequency, and no integration clusters were found in genes previously reported to link AAV and hepatocellular carcinoma development. ${ }^{128}$ Further assessment of risk will likely be an evolving theme as clinical trial data accumulates from next-generation AAV gene therapy vectors.

\section{SUMMARY}

AAV gene therapy for the liver has taken some time to come of age, with gene transfer research in hemophilia B as the forerunner of liver-directed gene therapy more broadly. Animal models for hemophilia B existed or were created, providing valid test subjects for therapeutic benefit. Assays existed for quantitative measurement of FIX, and the correlations of FIX level with therapeutic benefit in humans were well-established. The FIX gene is small, removing barriers for AAV vector engineering, and the field of mouse transgenics had provided basic information regarding control of gene transcription within hepatocytes. The stage was set for hemophilia B gene therapy proof-of-concept in animals, setting in motion the approach to human proof-of-concept for hemophilia B gene therapy. The final barriers that slowed progress to the clinic were cGMP vector manufacturing and the parallel regulatory requirements. With diligent work across the field, these barriers too were overcome.

Early clinical successes were limited, but importantly shed light on areas that required subsequent translational research, especially in the realm of immune responses to vector capsid proteins. While detailed hypotheses have been proposed as to mechanisms of the observed limitations of vector "expression," no iron-clad proof has been elucidated. This is not all that surprising, since the phenomena have not been recapitulated in animal models and the number of clinical examples is limited. One could conclude that advances in AAV vector capsid technology have likely played an important role in clinical successes. There is an enormous foundation of vectorology, animal science, and now clinical successes that potentially point to a brighter future for patients.

We should not be surprised nor sheepish about the duration of this journey to date. Consider the profound influence on medicine that could be wrought if gene therapy success was brought to an ever-increasing number of diseases. Today, there are multiple clinical trials underway in hemophilia and other liver-based disorders (see Table 1), as

Table 1. Ongoing and planned liver-directed AAV gene therapy clinical trials

\begin{tabular}{|c|c|c|c|c|}
\hline Trial Sponsor & Indication & AAV serotype & Stage & NCT Identifier \\
\hline BioMarin & Hemophilia A & AAV5 & Phase I/II & NCT02576795 \\
\hline \multirow[t]{4}{*}{ Dimension Therapeutics } & Hemophilia B & AAVrh10 & Phase I/II & NCT02618915 \\
\hline & OTC deficiency & AAV8 & IND-enabling & NA \\
\hline & GSDla & AAV8 & IND-enabling & NA \\
\hline & Wilson disease & $\mathrm{ND}$ & $\mathrm{DC}$ & NA \\
\hline Dimension/Bayer & Hemophilia A & ND & IND-enabling & NA \\
\hline Genethon & Crigler Najjar & ND & Preclinical & NA \\
\hline REGENXBIO/PENN & $\mathrm{HoFH}$ & AAV8 & Phase I/II & NCT02651675 \\
\hline Sangamo & Hemophilia A & AAV6 & IND-enabling & NA \\
\hline \multirow[t]{2}{*}{ Selecta Biosciences } & OTC deficiency & AAV & Preclinical & NA \\
\hline & Methylmalonic acidemia & Anc80 & Preclinical & NA \\
\hline Spark & Hemophilia A & ND & IND-enabling & NA \\
\hline Spark Therapeutics/CHOP & Hemophilia B & AAV8 & Phase $1 / \|$ & NCT01620801 \\
\hline UCL/SJCRH & Hemophilia B & AAV8 & Phase I/II & NCT00979238 \\
\hline uniQure/Chiesi & Hemophilia B & AAV5 & Phase $\mathrm{I} / \mathrm{II}$ & NCT02396342 \\
\hline uniOure & Hemophilia A & AAV5 & Preclinical & NA \\
\hline uniQure/Digna & AIP & AAV5 & Complete & NCT02082860 \\
\hline
\end{tabular}

ND, not disclosed; CIMA, Center for Applied Medical Research University of Navarra; HoFH, homozygous familial hypercholesterolemia; CHOP, Children's Hospital of Philadelphia; UCL/SCJRH, University College London/St. Jude Children's Research Hospital; OTC, ornithine transcarbamylase deficiency; GSDla, glycogen storage disease type la; PKU, phenylketonuria; AIP, acute intermittent porphyria; IND, investigational new drug; DC, development candidate; NA, not applicable.

Sources: company websites, clinicaltrials.gov, Thomson Cortellis 2016. 
well as other target organs. Definitive assessments of the results of ongoing clinical trials will have to await publication of results in peer-reviewed publications, but as a field we should expect to witness successes.

As we expand beyond the realm of secreted protein therapeutics characterized by hemophilia, additional aspects of liver gene transfer become important. For example, the importance of which hepatocytes undergo gene transfer becomes paramount. Due to metabolic zonation of the liver, hepatocytes proximal to the portal triad are active in metabolic processes such as the urea cycle, while those hepatocytes adjacent to the central vein are specialized for other functions. Thus, gene transfer to hepatocytes involved in metabolic syndromes is likely more critical than gene transfer across the liver. Endpoints for assessing success of gene transfer also become more challenging than the simple measurement of FIX in the blood. Fortunately, there are AAV vector capsids that do efficiently transduce metabolically active hepatocytes, and for many metabolic diseases, blood-based biomarkers and endpoints are already available.

We should not be surprised or overwhelmed by the opportunities for improvements across the field: vector design, manufacturing operations, adjunct therapies. This scenario would be expected and familiar given the field of hybridomas leading to therapeutic antibodies, where discoveries are still being made at a fast pace. The field is ripe for application of today's technology for today's therapies while pursuing technological improvements that may fuel expansion of gene therapies to new and more complex therapeutic indications. It is probably an apt paraphrase to say that we are now at "the end of the beginning" of this amazing and important enterprise.

\section{AUTHOR DISCLOSURE}

The authors declare that they have no potential competing financial interests. K.C. and S.W. are shareholders in Dimension Therapeutics. L.K., C.T., L.S., D.G., T.W., K.C., and S.W. are employees of Dimension Therapeutics.

\section{REFERENCES}

1. Mount JD, Herzog RW, Tillson DM, et al. Sustained phenotypic correction of hemophilia B dogs with a factor IX null mutation by liver-directed gene therapy. Blood 2002;99:2670-2676.

2. Snyder RO, Miao C, Meuse L, et al. Correction of hemophilia $B$ in canine and murine models using recombinant adeno-associated viral vectors. Nat Med 1999;5:64-70.

3. Wang L, Nichols TC, Read MS, et al. Sustained expression of therapeutic level of factor IX in hemophilia $B$ dogs by AAV-mediated gene therapy in liver. Mol Ther 2000;1:154-158.

4. Jiang $H$, Lillicrap D, Patarroyo-White $S$, et al. Multiyear therapeutic benefit of AAV serotypes 2,6 , and 8 delivering factor VIII to hemophilia A mice and dogs. Blood 2006;108:107-115.

5. Manno CS, Pierce GF, Arruda VR, et al. Successful transduction of liver in hemophilia by AAV-Factor IX and limitations imposed by the host immune response. Nat Med 2006;12:342-347.

6. Nathwani AC, Reiss UM, Tuddenham EG, et al. Long-term safety and efficacy of factor IX gene therapy in hemophilia B. N Engl J Med 2014;371: 1994-2004.

7. Gordillo M, Evans T, Gouon-Evans V. Orchestrating liver development. Development 2015;142:20942108.

8. Tortora GJ, Derrickson BH. The digestive system: liver and gallbladder. In: Tortora GJ, Derrickson $\mathrm{BH}$, eds. Principles of Anatomy and Physiology. Hoboken, NJ: John Wiley \& Sons, 2012:990-994.
9. Gissen P, Arias IM. Structural and functional hepatocyte polarity and liver disease. J Hepatol 2015;63:1023-1037.

10. Gruppuso PA, Sanders JA. Regulation of liver development: implications for liver biology across the lifespan. J Mol Endocrinol 2016;56:R115-125.

11. Fagiuoli $S$, Daina E, D'Antiga $L$, et al. Monogenic diseases that can be cured by liver transplantation. J Hepatol 2013;59:595-612.

12. Alam S, Sood V. Metabolic liver disease: when to suspect and how to diagnose? Indian $\mathrm{J} \mathrm{Pe}$ diatr 2016;83:1321-1333.

13. Orphanet.

14. Macdonald RA. "Lifespan" of liver cells. Autoradio-graphic study using tritiated thymidine in normal, cirrhotic, and partially hepatectomized rats. Arch Intern Med 1961;107:335-343.

15. Terpstra OT, Malt RA, Bucher NL. Negligible role of adrenal hormones in regulation of DNA synthesis in livers of partially hepatectomized rats. Proc Soc Exp Biol Med 1979;161:326-331.

16. Duncan AW, Dorrell C, Grompe M. Stem cells and liver regeneration. Gastroenterology 2009;137: 466-481.

17. Online Mendelian Inheritance in Man (OMIM)

18. Jacobs F, Gordts SC, Muthuramu I, et al. The liver as a target organ for gene therapy: state of the art, challenges, and future perspectives. Pharmaceuticals (Basel) 2012;5:1372-1392.
19. Vollmar B, Menger MD. The hepatic microcirculation: mechanistic contributions and therapeutic targets in liver injury and repair. Physiol Rev 2009;89:1269-1339.

20. Gao G, Alvira MR, Somanathan S, et al. Adenoassociated viruses undergo substantial evolution in primates during natural infections. Proc Natl Acad Sci U S A 2003;100:6081-6086.

21. Gao G, Vandenberghe LH, Alvira MR, et al. Clades of Adeno-associated viruses are widely disseminated in human tissues. J Virol 2004;78:6381-6388.

22. Grisham JW. A morphologic study of deoxyribonucleic acid synthesis and cell proliferation in regenerating rat liver; autoradiography with thymidine-H3. Cancer Res 1962;22:842-849.

23. Nienhuis AW, Nathwani AC, Davidoff AM. Gene therapy for hemophilia. Hum Gene Ther 2016;27: 305-308.

24. Bickel H, Gerrard J, Hickmans EM. Influence of phenylalanine intake on phenylketonuria. Lancet 1953;265:812-813.

25. Chapman MS, Agbandje-McKenna M. Atomic structure of viral particles. In: Kerr JR, Cotmore SF, Bloom ME, Linden RM, Parrish CR, eds. The Parvoviruses. London: Hodder Arnold, 2006:107-123.

26. Ou W, Wang M, Wu Y, et al. Scalable downstream strategies for purification of recombinant adenoassociated virus vectors in light of the properties. Curr Pharm Biotechnol 2015;16:684-695

27. Burova E, loffe E. Chromatographic purification of recombinant adenoviral and adeno-associated 
viral vectors: methods and implications. Gene Ther 2005;12:S5-17.

28. Clement N, Grieger JC. Manufacturing of recombinant adeno-associated viral vectors for clinical trials. Mol Ther Methods Clin Dev 2016;3:16002.

29. Berns Kl, Giraud C. Adenovirus and adenoassociated virus as vectors for gene therapy. Ann N Y Acad Sci 1995;772:95-104.

30. Dong B, Nakai H, Xiao W. Characterization of genome integrity for oversized recombinant AAV vector. Mol Ther 2010:18:87-92.

31. Wu Z, Yang H, Colosi P. Effect of genome size on AAV vector packaging. Mol Ther 2010;18:80-86.

32. Chamberlain K, Riyad JM, Weber T. Expressing transgenes that exceed the packaging capacity of adeno-associated virus capsids. Hum Gene Ther Methods 2016;27:1-12.

33. Dong JY, Fan PD, Frizzell RA. Quantitative analysis of the packaging capacity of recombinant adenoassociated virus. Hum Gene Ther 1996;7:2101-2112.

34. Grieger JC, Samulski RJ. Packaging capacity of adeno-associated virus serotypes: impact of larger genomes on infectivity and postentry steps. J Virol 2005;79:9933-9944.

35. Lind P, Larsson K, Spira J, et al. Novel forms of B-domain-deleted recombinant factor VIII molecules. Construction and biochemical characterization. Eur J Biochem 1995;232:19-27.

36. Hirata RK, Russell DW. Design and packaging of adeno-associated virus gene targeting vectors. J Virol 2000;74:4612-4620.

37. Gao GP, Alvira MR, Wang L, et al. Novel adenoassociated viruses from rhesus monkeys as vectors for human gene therapy. Proc Natl Acad Sci U S A 2002;99:11854-11859.

38. Zincarelli C, Soltys S, Rengo G, et al. Analysis of AAV serotypes 1-9 mediated gene expression and tropism in mice after systemic injection. Mol Ther 2008;16:1073-1080.

39. Wang L, Wang H, Bell P, et al. Systematic evaluation of $A A V$ vectors for liver directed gene transfer in murine models. Mol Ther 2010;18:118-125.

40. Vandenberghe $L H$, Xiao $R$, Lock $M$, et al. Efficient serotype-dependent release of functional vector into the culture medium during adeno-associated virus manufacturing. Hum Gene Ther 2010;21:12511257.

41. Calcedo R, Vandenberghe LH, Gao G, et al. Worldwide epidemiology of neutralizing antibodies to adeno-associated viruses. $\mathrm{J}$ Infect Dis 2009;199:381-390.

42. Zhong L, Li B, Mah CS, et al. Next generation of adeno-associated virus 2 vectors: point mutations in tyrosines lead to high-efficiency transduction at lower doses. Proc Natl Acad Sci U S A 2008;105: 7827-7832.

43. Cheng B, Ling C, Dai $Y$, et al. Development of optimized AAV3 serotype vectors: mechanism of high-efficiency transduction of human liver cancer cells. Gene Ther 2012;19:375-384.
44. Raupp C, Naumer M, Muller OJ, et al. The threefold protrusions of adeno-associated virus type 8 are involved in cell surface targeting as well as postattachment processing. J Virol 2012;86:93969408.

45. Warrington KH Jr, Gorbatyuk OS, Harrison JK, et al. Adeno-associated virus type 2 VP2 capsid protein is nonessential and can tolerate large peptide insertions at its $\mathrm{N}$ terminus. J Virol 2004;78:6595-6609.

46. Munch RC, Janicki H, Volker I, et al. Displaying high-affinity ligands on adeno-associated viral vectors enables tumor cell-specific and safe gene transfer. Mol Ther 2013;21:109-118.

47. Grimm D, Lee JS, Wang $L$, et al. In vitro and in vivo gene therapy vector evolution via multispecies interbreeding and retargeting of adenoassociated viruses. J Virol 2008;82:5887-5911.

48. Lisowski L, Dane AP, Chu K, et al. Selection and evaluation of clinically relevant AAV variants in a xenograft liver model. Nature 2014;506:382-386.

49. Kuriyama S, Yoshikawa M, Ishizaka S, et al. A potential approach for gene therapy targeting hepatoma using a liver-specific promoter on a retroviral vector. Cell Struct Funct 1991;16:503-510

50. Schiedner G, Morral N, Parks RJ, et al. Genomic DNA transfer with a high-capacity adenovirus vector results in improved in vivo gene expression and decreased toxicity. Nat Genet 1998;18:180-183.

51. Dang 0 , Walker D, Taylor S, et al. Structure of the hepatic control region of the human apolipoprotein E/C-I gene locus. J Biol Chem 1995;270:2257722585.

52. Davidoff AM, Gray JT, Ng CY, et al. Comparison of the ability of adeno-associated viral vectors pseudotyped with serotype 2, 5, and 8 capsid proteins to mediate efficient transduction of the liver in murine and nonhuman primate models. Mol Ther 2005;11: 875-888.

53. Hafenrichter DG, Wu X, Rettinger SD, et al. Quantitative evaluation of liver-specific promoters from retroviral vectors after in vivo transduction of hepatocytes. Blood 1994;84:3394-3404.

54. Miao CH, Ohashi K, Patijn GA, et al. Inclusion of the hepatic locus control region, an intron, and untranslated region increases and stabilizes hepatic factor IX gene expression in vivo but not in vitro. Mol Ther 2000;1:522-532.

55. McIntosh J, Lenting PJ, Rosales C, et al. Therapeutic levels of FVIII following a single peripheral vein administration of rAAV vector encoding a novel human factor VIII variant. Blood 2013;121: 3335-3344.

56. Nathwani AC, Gray JT, Ng CY, et al. Selfcomplementary adeno-associated virus vectors containing a novel liver-specific human factor IX expression cassette enable highly efficient transduction of murine and nonhuman primate liver. Blood 2006;107:2653-2661.

57. Hayashi $Y$, Mori $Y$, Janssen $O E$, et al. Human thyroxine-binding globulin gene: complete sequence and transcriptional regulation. Mol Endocrinol 1993;7:1049-1060.
58. Rouet $\mathrm{P}$, Raguenez $\mathrm{G}$, Tronche F, et al. A potent enhancer made of clustered liver-specific elements in the transcription control sequences of human alpha 1-microglobulin/bikunin gene. J Biol Chem 1992:267:20765-20773.

59. Wang L, Calcedo R, Nichols TC, et al. Sustained correction of disease in naive and AAV2-pretreated hemophilia B dogs: AAV2/8-mediated, liverdirected gene therapy. Blood 2005;105:3079-3086.

60. Wang L, Takabe K, Bidlingmaier SM, et al. Sustained correction of bleeding disorder in hemophilia B mice by gene therapy. Proc Natl Acad Sc U S A 1999;96:3906-3910.

61. Vigna E, Amendola M, Benedicenti F, et al. Efficient Tet-dependent expression of human factor IX in vivo by a new self-regulating lentiviral vector. Mol Ther 2005;11:763-775.

62. Chuah MK, Petrus I, De Bleser P, et al. Liverspecific transcriptional modules identified by genome-wide in silico analysis enable efficient gene therapy in mice and non-human primates. Mol Ther 2014;22:1605-1613.

63. Niemeyer GP, Herzog RW, Mount J, et al. Longterm correction of inhibitor-prone hemophilia $B$ dogs treated with liver-directed AAV2-mediated factor IX gene therapy. Blood 2009;113:797-806.

64. Dominguez $\mathrm{E}$, Marais T, Chatauret $\mathrm{N}$, et al. Intravenous scAAV9 delivery of a codon-optimized SMN1 sequence rescues SMA mice. Hum Mol Genet 2011;20:681-693.

65. Foster H, Sharp PS, Athanasopoulos T, et al. Codon and mRNA sequence optimization of microdystrophin transgenes improves expression and physiological outcome in dystrophic mdx mice following AAV2/8 gene transfer. Mol Ther 2008;16: 1825-1832.

66. Nathwani AC, Tuddenham EG, Rangarajan S, et al. Adenovirus-associated virus vector-mediated gene transfer in hemophilia B. N Engl J Med 2011;365: 2357-2365.

67. Paneda A, Lopez-Franco E, Kaeppel C, et al. Safety and liver transduction efficacy of rAAV5-cohPBGD in nonhuman primates: a potential therapy for acute intermittent porphyria. Hum Gene Ther 2013; 24:1007-1017.

68. Wang L, Morizono H, Lin J, et al. Preclinical evaluation of a clinical candidate AAV8 vector for ornithine transcarbamylase (OTC) deficiency reveals functional enzyme from each persisting vector genome. Mol Genet Metab 2012;105:203-211.

69. Hunt RC, Simhadri VL, landoli M, et al. Exposing synonymous mutations. Trends Genet 2014;30:308321.

70. Marin M. Folding at the rhythm of the rare codon beat. Biotechnol J 2008;3:1047-1057.

71. Wright JF. Manufacturing and characterizing AAV-based vectors for use in clinical studies. Gene Ther 2008;15:840-848.

72. Thorne BA, Takeya RK, Peluso RW. Manufacturing recombinant adeno-associated viral vectors from producer cell clones. Hum Gene Ther 2009;20:707714. 
73. Kotin RM. Large-scale recombinant adenoassociated virus production. Hum Mol Genet 2011;20:R2-6.

74. Hauswirth WW, Aleman TS, Kaushal S, et al. Treatment of leber congenital amaurosis due to RPE65 mutations by ocular subretinal injection of adeno-associated virus gene vector: short-term results of a phase I trial. Hum Gene Ther 2008;19: 979-990.

75. Grieger JC, Soltys SM, Samulski RJ. Production of recombinant adeno-associated virus vectors using suspension HEK293 cells and continuous harvest of vector from the culture media for GMP FIX and FLT1 clinical vector. Mol Ther 2016;24:287-297.

76. Thomas DL, Wang L, Niamke J, et al. Scalable recombinant adeno-associated virus production using recombinant herpes simplex virus type 1 coinfection of suspension-adapted mammalian cells. Hum Gene Ther 2009;20:861-870.

77. Clement N, Knop DR, Byrne BJ. Large-scale adenoassociated viral vector production using a herpesvirus-based system enables manufacturing for clinical studies. Hum Gene Ther 2009;20:796806 .

78. Martin J, Frederick A, Luo Y, et al. Generation and characterization of adeno-associated virus producer cell lines for research and preclinical vector production. Hum Gene Ther Methods 2013;24:253269.

79. Gao G, Lu Y, Calcedo R, et al. Biology of AAV serotype vectors in liver-directed gene transfer to nonhuman primates. Mol Ther 2006;13:77-87.

80. Nathwani AC, Cochrane M, Mclntosh J, et al. Enhancing transduction of the liver by adenoassociated viral vectors. Gene Ther 2009;16:60-69.

81. Bell $P$, Wang L, Gao G, et al. Inverse zonation of hepatocyte transduction with AAV vectors between mice and non-human primates. Mol Genet Metab 2011;104:395-403.

82. Cunningham SC, Dane AP, Spinoulas A, et al. Gene delivery to the juvenile mouse liver using AAV2/8 vectors. Mol Ther 2008;16:1081-1088.

83. Dane AP, Wowro SJ, Cunningham SC, et al Comparison of gene transfer to the murine liver following intraperitoneal and intraportal delivery of hepatotropic AAV pseudo-serotypes. Gene Ther 2013;20:460-464.

84. Alexander IE, Cunningham SC, Logan GJ, et al. Potential of AAV vectors in the treatment of metabolic disease. Gene Ther 2008;15:831-839.

85. Colnot S, Perret C. Liver zonation. In: Monga SPS, Cagle, PT eds. Molecular Pathology of Liver Diseases. New York, NY: Springer US, 2010:7-16.

86. Jiang $H$, Couto LB, Patarroyo-White $S$, et al. Effects of transient immunosuppression on adenoassociated, virus-mediated, liver-directed gene transfer in rhesus macaques and implications for human gene therapy. Blood 2006;108: 3321-3328.

87. Murphy SL, Li H, Zhou S, et al. Prolonged susceptibility to antibody-mediated neutralization for adeno-associated vectors targeted to the liver. $\mathrm{Mol}$ Ther 2008;16:138-145.

88. Li H, Murphy SL, Giles-Davis W, et al. Pre-existing AAV capsid-specific CD8+ T cells are unable to eliminate AAV-transduced hepatocytes. Mol Ther 2007;15:792-800.

89. Wang L, Figueredo J, Calcedo R, et al. Crosspresentation of adeno-associated virus serotype 2 capsids activates cytotoxic $T$ cells but does not render hepatocytes effective cytolytic targets. Hum Gene Ther 2007;18:185-194.

90. Basner-Tschakarjan E, Bijjiga E, Martino AT. Preclinical assessment of immune responses to adeno-associated virus (AAV) vectors. Front Immunol 2014;5:28.

91. Li C, Hirsch M, DiPrimio N, et al. Cytotoxic-Tlymphocyte-mediated elimination of target cells transduced with engineered adeno-associated virus type 2 vector in vivo. J Virol 2009;83:6817-6824.

92. Nguyen DH, Hurtado-Ziola N, Gagneux P, et al. Loss of Siglec expression on $T$ lymphocytes during human evolution. Proc Natl Acad Sci U S A 2006;103:7765-7770.

93. Pastore L, Morral N, Zhou H, et al. Use of a liverspecific promoter reduces immune response to the transgene in adenoviral vectors. Hum Gene Ther 1999;10:1773-1781.

94. Cao O, Dobrzynski E, Wang L, et al. Induction and role of regulatory $\mathrm{CD} 4+\mathrm{CD} 25+\mathrm{T}$ cells in tolerance to the transgene product following hepatic in vivo gene transfer. Blood 2007;110:1132-1140.

95. Mingozzi F, Hasbrouck NC, Basner-Tschakarjan E, et al. Modulation of tolerance to the transgene product in a nonhuman primate model of AAVmediated gene transfer to liver. Blood 2007; 110:2334-2341.

96. Limmer A, Ohl J, Kurts C, et al. Efficient presentation of exogenous antigen by liver endothelial cells to $C D 8+T$ cells results in antigen-specific $T$ cell tolerance. Nat Med 2000;6:1348-1354.

97. Horst AK, Neumann K, Diehl L, et al. Modulation of liver tolerance by conventional and nonconventional antigen-presenting cells and regulatory immune cells. Cell Mol Immunol 2016;13:277-292.

98. Crispe IN. Hepatic T cells and liver tolerance. Nat Rev Immunol 2003;3:51-62.

99. Crispe IN. Immune tolerance in liver disease. Hepatology 2014;60:2109-2117.

100. Mingozzi F, Liu YL, Dobrzynski E, et al. Induction of immune tolerance to coagulation factor IX antigen by in vivo hepatic gene transfer. J Clin Invest 2003;111:1347-1356.

101. LoDuca PA, Hoffman BE, Herzog RW. Hepatic gene transfer as a means of tolerance induction to transgene products. Curr Gene Ther 2009;9: 104-114.

102. Sun JY, Anand-Jawa V, Chatterjee S, et al. Immune responses to adeno-associated virus and its recombinant vectors. Gene Ther 2003;10: 964-976.
103. Sack BK, Merchant S, Markusic DM, et al Transient B cell depletion or improved transgene expression by codon optimization promote tolerance to factor VIII in gene therapy. PloS One 2012;7:e37671.

104. Herzog RW, Mount JD, Arruda VR, et al. Muscledirected gene transfer and transient immune suppression result in sustained partial correction of canine hemophilia B caused by a null mutation. Mol Ther 2001;4:192-200.

105. Fields PA, Arruda VR, Armstrong E, et al. Risk and prevention of anti-factor IX formation in AAV-mediated gene transfer in the context of a large deletion of F9. Mol Ther 2001:4:201-210.

106. Ellinwood NM, Ausseil J, Desmaris N, et al. Safe, efficient, and reproducible gene therapy of the brain in the dog models of Sanfilippo and Hurler syndromes. Mol Ther 2011;19:251-259.

107. Messinger YH, Mendelsohn NJ, Rhead W, et al. Successful immune tolerance induction to enzyme replacement therapy in CRIM-negative infantile Pompe disease. Genet Med 2012;14: 135-142.

108. Joly MS, Martin RP, Mitra-Kaushik S, et al. Transient low-dose methotrexate generates $B$ regulatory cells that mediate antigen-specific tolerance to alglucosidase alfa. J Immunol 2014; 193:3947-3958

109. Moskalenko M, Chen L, van Roey M, et al. Epitope mapping of human anti-adeno-associated virus type 2 neutralizing antibodies: implications for gene therapy and virus structure. J Virol 2000;74:1761-1766.

110. Calcedo R, Wilson JM. Humoral Immune Response to AAV. Front Immunol 2013;4:341.

111. Calcedo R, Wilson JM. AAV natural infection induces broad cross-neutralizing antibody responses to multiple AAV serotypes in chimpanzees. Hum Gene Ther Clin Dev 2016;27:79-82.

112. Chicoine LG, Montgomery CL, Bremer WG, et al. Plasmapheresis eliminates the negative impact of AAV antibodies on microdystrophin gene expression following vascular delivery. Mol Ther 2014;22:338-347.

113. Mingozzi F, Anguela XM, Pavani G, et al. Overcoming preexisting humoral immunity to AAV using capsid decoys. Sci Transl Med 2013;5: 194ra192.

114. Donsante A, Vogler C, Muzyczka N, et al. Observed incidence of tumorigenesis in long-term rodent studies of rAAV vectors. Gene Ther 2001:8:1343-1346

115. Donsante A, Miller DG, Li Y, et al. AAV vector integration sites in mouse hepatocellular carcinoma. Science 2007;317:477.

116. Chandler RJ, LaFave MC, Varshney GK, et al. Vector design influences hepatic genotoxicity after adeno-associated virus gene therapy. J Clin Invest 2015;125:870-880.

117. Luk JM, Burchard J, Zhang C, et al. DLK1-DIO3 genomic imprinted microRNA cluster at $14 q 32.2$ 
defines a stemlike subtype of hepatocellular carcinoma associated with poor survival. J Biol Chem 2011;286:30706-30713.

118. Bell P, Moscioni AD, McCarter RJ, et al. Analysis of tumors arising in male B6C3F1 mice with and without AAV vector delivery to liver. Mol Ther 2006;14:34-44.

119. Zhong L, Malani N, Li M, et al. Recombinant adeno-associated virus integration sites in murine liver after ornithine transcarbamylase gene correction. Hum Gene Ther 2013;24:520525.

120. Li H, Malani N, Hamilton SR, et al. Assessing the potential for AAV vector genotoxicity in a murine model. Blood 2011;117:3311-3319.
121. Nault JC, Datta $S$, Imbeaud $S$, et al. Recurrent AAV2-related insertional mutagenesis in human hepatocellular carcinomas. Nat Genet 2015;47: 1187-1193.

122. Berns KI, Byrne BJ, Flotte TR, et al. Adenoassociated virus type 2 and hepatocellular carcinoma? Hum Gene Ther 2015;26:779-781.

123. Buning $H$, Schmidt M. Adeno-associated vector toxicity — to be or not to be? Mol Ther 2015;23: 1673-1675.

124. Smith RH. Adeno-associated virus integration: virus versus vector. Gene Ther 2008;15:817-822.

125. Naldini L. Gene therapy returns to centre stage. Nature 2015;526:351-360.
126. Gene Therapy Clinical Trials Worldwide. Provided by The Journal of Gene Medicine, Wiley. www.abedia .com/wiley/ (last accessed December 12, 2016).

127. Kaeppel C, Beattie SG, Fronza R, et al. A largely random AAV integration profile after LPLD gene therapy. Nat Med 2013;19:889-891.

128. Gil-Farina I, Fronza R, Kaeppel C, et al. Recombinant AAV integration is not associated with hepatic genotoxicity in nonhuman primates and patients. Mol Ther 2016;24:1100-1105.

Received for publication October 31, 2016; accepted after revision November 23, 2016.

Published online: November 28, 2016. 